\title{
AN LC- MS/MS METHOD FOR THE DETERMINATION OF OMEPRAZOLE ON PROTON PUMP INHIBITOR IN HUMAN PLASMA
}

\section{T. Sudha*, V. Kalan Kumar Reddy ${ }^{1}$, P.V. Hemalatha ${ }^{2}$, V.R. Ravikumar ${ }^{3}$}

\begin{abstract}
${ }^{1}$ Dept Pharma. Analysis, The Erode College of Pharmacy, Erode TamilNadu, India. 638112.

2Dept Pharm. Chemistry, The Erode College of Pharmacy, Erode TamilNadu, India. 638112.

${ }^{3}$ Dept Pharmacognosy, The Erode College of Pharmacy, Erode, TamilNadu, India. 638112.
\end{abstract}

Submitted: 09-02-2016

Revised: $13-03-2016$

Accepted: $02-04-2016$

*Corresponding author

T. Sudha

Email:

jvchrsty@yahoo.co.in

\section{ABSTRACT}

A sensitive and selective liquid chromatographic method coupled with tandem mass spectroscopy (LC-MS/MS) was developed for the quantification of omeprazole in human plasma. Lansoprazole was used as internal standard with plasma samples, extracted using $10 \mathrm{mM}$ ammonium acetate. A centrifuged upper layer was then evaporated and reconstituted with Acetonitrile: mobile phase buffer $70: 30 \% \mathrm{v} / \mathrm{v}$. The reconstructed samples were injected into a $\mathrm{C}_{18}$ column purospher star $5 \mu$. The mobile phase was composed of ACN: mobile phase buffer $(5 \mathrm{~mm}$ ammonium bicarbonate buffer) in the ratio of $70: 30 \% \mathrm{v} / \mathrm{v}$ with flow rate $1.0 \mathrm{~mL} / \mathrm{min}$. The mass spectrometer was operated using positive ion mode and turbo electro spray ionisation. Nitrogen was used as the nebulizer, curtain, collision and auxiliary gases. Using MS/MS with multiple reactions monitoring (MRM) mode, omeprazole was detected without severe interferences from plasma matrix. Detection of omeprazole in human plasma was accurate and precision. This method has been successfully applied to the study of omeprazole in human specimens

Keywords: Proton pump inhibitor, omeprazole, lansoprazole, LC-MS/MS, liquid liquid extraction

\section{INTRODUCTION}

Omeprazole is chemically known as $1 H$ Benzimidazole,5-methoxy-2-[[(4-methoxy-3,5dimethyl-2pyridinyl)methyl] sulfinyl]-.5-Methoxy2-[[(4-methoxy-3,5-dimethyl-2pyridinyl)methyl $]$ sulfinyl] benzimidazole. Lansoprazole (internal standard) is chemically known as (Figure-1) (RS) -2- ([3 - methyl-4-(2, 2, 2 - trifluoromethoxy) pyridine-2-yl] methyl sulfinyl) $-1 \mathrm{H}$ - benzo[d] imidazole. Omeprazole is a selective and irreversible proton pump inhibitor. Omeprazole suppresses gastric acid secretion by specific inhibition of the hydrogen-potassium adenosine triphosphatase $\left(\mathrm{H}^{+} \mathrm{K}^{+}\right.$-ATPase $)$enzyme system found at the secretory surface of parietal cells. It inhibits the final transport of hydrogen ions (via exchange with potassium ions) into the gastric lumen. Since the $\mathrm{H}^{+} \mathrm{K}^{+}$-ATPase enzyme system is regarded as the acid (proton) pump of the gastric mucosa. Omeprazole is known as a gastric acid pump inhibitor. Omeprazole is also used to treat infection caused by Helicobacter pylori (H. pylori). Recently, cytochrome p-450 2C19 genotype related anti-H. pylori efficacy by combining omeprazole and antibiotics were reported (Rang et al., 2007 and Furuta et al., 2007). Determination of the omeprazole concentration in body fluids including serum, plasma and cerebrospinal fluid is of importance in conducting clinical studies of this drug with regard to efficacy, toxicity and dose ranging.<smiles>COc1ccc2[nH]c(S(=O)Cc3ncc(C)c(OC)c3C)nc2c1</smiles><smiles>Cc1c(OCC(F)(F)F)ccnc1CS(=O)c1nc2ccccc2[nH]1</smiles>

Lansoprazole

Figure 1. Structure for omeprazole and lansoprazole 
Modern pharmacokinetic studies require parts per billion characterizations and quantification as well as the ability to provide analytical results with rapid turnaround from large batches of samples. However, in recent years the use of high performance liquid chromatography (HPLC) with ultra violet (UV) detection, mass spectrometric detection (MSD) and tandem mass spectrometry (MS-MS) have been found to be ideally suited for the determination of analyte in diverse biometrics. Determination of omeprazole and its main metabolites by liquid chromatography using hybrid miceller mobile phase (Rambala, et al., 2009). Enantiomers of omeprazole in blood plasma were determined by normal-phase liquid chromatography and detected using atmospheric pressure ionization tandem mass spectrometry (Stenhoff et al., 1999). Chiral assay of omeprazole and metabolites and its application to a pharmacokinetics related to CYP2C19 genotypes (Shiohira et al., 2011). Assay of omeprazole and omeprazole sulfone has been reported using semi-micro column liquid chromatography with mixed-function precolumn (Yim et al., 2001). Several HPLC methods have been published for quantitatively determining omeprazole levels in human plasma (Vittal et al, 2009; DeSmet et al., 2010; TsingHua 2004; Bharathi et al., 2009; Kanazawa et al., 2004; Wang et al., 2004 and Kang et al., 1999). The methods are isocratic and gradient HPLC (or) HPLC with column switching liquid chromatography mass spectrometry (LC-MS/MS). Some of these assay methods are relatively time consuming while others have poor lower limit of quantitation. Sample preparation by liquid liquid extraction was another time consuming step in these methods. Therefore, our goal is to develop a relatively rapid, high sensitivity method for omeprazole in human plasma with very low limit of quantification using LCMS/MS instrument. This paper discusses the method validation of rapid, liquid chromatography tandem mass spectrometric (LCMS/MS) assay for omeprazole in human plasma using lansoprazole as internal standard.

The sample preparation is simple and consists of precipitating plasma with $10 \mathrm{~mm}$ extraction buffer ammonium acetate (pH 10.0) and $2 \mathrm{~mL}$ TBME solvent by vortex mixing and spinning down the protein into a pellet. The supernatant is injected into a LC column and eluted with isocratic of $5 \mathrm{~mm}$ ammonium bicarbonate buffer ( $\mathrm{pH}$ 8.0) and Acetonitrile. A board calibration curve range from 5.016 to 4013.028ng/mL was adequate to handle most pharmacokinetic samples. Some samples were obtained at extended sampling intervals beyond the $12 \mathrm{~h}$ and also to measure samples from peak concentrations without dilution. However, these published methods are not ideal for pharmacokinetic work, because they are time consuming i.e., derivatization step, arduous sample preparation and long chromatographic run time. Likewise, they need a relatively large amount of sample to reach a low quantification limit. In addition detection of omeprazole using LC-MS/MS has to be reported.

Therefore, this study established a novel quantification method for detecting omeprazole in human plasma using liquid chromatography electron spray tandem mass spectroscopy. This method has been successfully applied to pharmacokinetic studies to determine the concentration of omeprazole in human plasma.

\section{MATERIAL AND METHODS Chemicals and reagents}

All solvents including HPLC grade methanol, acetonitrile and tertiary butyl methyl ether were obtained from Rankem, Mumbai. Ammonia solution (SQ grade 25\%), sodium bicarbonate (GR grade 99.5\%), ammonium bicarbonate (HR grade 99\%), ammonium acetate (GR grade 98\%) were also obtained from Rankem Mumbai. Aurobindho Pharmaceuticals provided both Omeprazole, Internal standard (Lansoprazole) and ethylene diamine tetra acetic acid (EDTA) treated plasma sample. A Millipore Milli-Q Plus was used to generate deionized water inhouse.

\section{Instrumentation}

Water 2695 HPLC coupled with a Quattro premix XE mass spectrometer was utilized for the separation and detection of Omeprazole. Both HPLC and mass spectrometer were controlled remotely using Mass Lynx software V 4.1. Data analysis was performed with the QuanLynx module that accompanies Mass Lynx. 


\section{Calibration standard and quality control preparation}

A $1 \mathrm{mg} / \mathrm{mL}$ master stock solution of Omeprazole was prepared in $7: 3 \mathrm{v} / \mathrm{v}$ ACN: mobile phase buffer (5Mm ammonium bicarbonate buffer 8.0). This solution was diluted to make a series of standard working solutions at 5,10,40,80,200,400,1000,2000,3200 and $4000 \mathrm{ng} / \mathrm{mL}$. A $100 \mu \mathrm{g} / \mathrm{mL}$ stock solution of internal standard (Lansoprazole) was prepared in $7: 3 \mathrm{v} / \mathrm{v} A C N$ : mobile phase buffer and diluted to $1 \mu \mathrm{g} / \mathrm{mL}$. For preparation of calibration curves, $10 \mu \mathrm{L}$ of appropriate levels of calibration standard and $10 \mu \mathrm{L}$ of Lansoprazole (IS) was added to $200 \mu \mathrm{L}$ of freshly thawed EDTA plasma in a borosilicate glass culture tube, and mixed. A separate weighing was used in order to prepare $1 \mathrm{mg} / \mathrm{mL}$ quality control master stock solution in 7:3ACN: mobile phase buffer. The quality control master stock solution was diluted to 256.43 , $732.672, \quad 6912.00, \quad 17280.00,86400.00$ and $160000.00 \mathrm{ng} / \mathrm{mL}$ for preparing lower limit, lower, intermediate, medium 1 , medium 2 and high -level quality control working solutions. Plasma quality controls were prepared by addition of $0.2 \mathrm{~mL}$ of the appropriate level of quality control working solution to $9.8 \mathrm{~mL}$ of EDTA plasma, for final concentrations of 5, $14.653,345.600,1728.00,3200.00 \mathrm{ng} / \mathrm{mL}$. All master solutions, working standards and quality control standards were stored in refrigerator at $2-8^{\circ} \mathrm{C}$.

\section{Sample preparation}

Calibration standards were prepared as described above. Additional samples consisted of $200 \mu \mathrm{L}$ of unknown or quality control plasma. Internal standard and 7:3-ACN: mobile phase buffer were added to all unknown and quality control samples. Blank (plasma only with 7:3 ACN: mobile phase buffer, no internal standard) and $100 \mu \mathrm{L}$ of plasma sample with internal standard and $20 \mu \mathrm{L}$ ACN: mobile phase. Samples were included in every run. An adaptation of a previously published liquid liquid extraction was employed in order to separate Omeprazole from plasma components (Wang et al., 2004). All calibration standards, quality control samples, and unknown samples were alkalised by the addition of an equal volume of $10 \mathrm{mM}$ ammonium acetate buffer $\mathrm{pH}$
10.0, and then mixed by vortexing in a glass culture tubes. Omeprazole was extracted by an addition of $2 \mathrm{~mL}$ of tertiary butyl methyl ether and samples were thoroughly mixed by vortexing for a period of $15 \mathrm{~min}$ in rotospin at 40rpm. Then centrifugation was done for $4500 \mathrm{rpm}$ at $40^{\circ} \mathrm{C}$ for $5 \mathrm{~min}$. Following centrifugation, the aqueous component was frozen in a dry ice isopropanol bath and the organic component was decanted into a separate $2.0 \mathrm{~mL}$ micro centrifuge tube. Organic phase was evaporated under a stream of nitrogen in $30 \pm 5^{\circ} \mathrm{C}$ water bath using a TurboVap LV concentration work station. Dried samples were reconstituted to $500 \mu \mathrm{L}$ using reconstituted solution and mixed by vortexing for about $1 \mathrm{~min}$. $10 \mu \mathrm{L}$ sample solution were then transferred to low volume inserts in HPLC vials and loaded into the autosampler.

\section{HPLC separation and MS-MS condition}

All samples were subjected to separation using a Water 2695 HPLC with a Purospher Star $\mathrm{C}_{18}$ column $(5 \mu, 100 \mathrm{X} 4.6 \mathrm{~mm})$. Separation was achieved with an isocratic run using mobile phase consisted of a mixture of acetonitrile with $5 \mathrm{mM}$ ammonium bicarbonate buffer $(7: 3 \% \mathrm{v} / \mathrm{v})$. The $\mathrm{pH}$ of the mobile phase was then adjusted to 8.0 with formic acid. Injection consisted of $10 \mu \mathrm{L}$ of each sample, the flow was set at $1.0 \mathrm{~mL} / \mathrm{min}$ and the overall run time was 2.0min. Omeprazole and internal standard (lansoprazole) were detected as they eluted from the column using MS-MS detection in the electrospray positive $\left(\mathrm{ES}^{+}\right)$mode. The first Quadra pole $\mathrm{Q}_{1}$ was set to monitor the protonated molecule $(\mathrm{m}+1)$ at $\mathrm{m} / \mathrm{z} 346.18$ and 369.97 for omeprazole and internal standard respectively. Product ions resulting from collision induced fragmentation at $\mathrm{Q}_{2}$ were monitored via $\mathrm{Q}_{3}$ at $\mathrm{m} / \mathrm{z} 198.00$ and 250.00 for omeprazole and internal standard respectively (Table I).

\section{Bioanalytical method validation}

A through and complete method validation in human plasma was done following the U.S.FDA guidelines.

Validation runs containing the full calibration curve, blank samples, six replicates each of the lower limit of quantification 
(LLOQ 5ng/mL) low, mid and high level quality control samples were run on six different days. Six different lots of EDTA treated human plasma were used during the validation process.

The carryover effect of the auto sampler was evaluated by sequentially injecting solutions of analytes (aqueous standard), reconstitution solution, standard blank and extracted standards of analytes, equivalent to highest standard in the calibration range.

The calibration procedure is based on the use of various standards, often prepared and measured in duplicate, for the assessment of the calibration model. The use of pure synthetic solutions that can be used for the preparation of standards is limited to those methods without matrix effects (Antonio Checa et al). Calibration models are relying on linear relationship between responses and concentration. Calibration standards in plasma sample containing 5.0 to $4000.00 \mathrm{ng} / \mathrm{mL}$ were used to establish a single calibration curve with $1 / \mathrm{X}^{2}$ weighed linear equation. Lowest limit of quantification (LLOQ) of drug as the minimal concentration in the samples could be determined with a deviation lower than $\pm 20 \%$. Concentrations of LLOQ and quality control samples were determined from calibration curves created with each run and at least four out of six of these had be within $15 \%$ of nominal value.

The selectivity / specificity of the method towards endogenous plasma matrix components, metabolites and concomitant medications was assessed in ten batches ( 7 normal of $\mathrm{K}_{2}$ EDTA, 1 heamolyzed, 1 lipidemic and 1 heparinized) of blank human plasma. Cross talk of MRM channels for analyte and IS were checked using the highest concentration from the calibration curve and the working solution of IS. The effect of potential interfering drugs like paracetamol, ibuprofen, caffeine, diphenyl hydramine hydrochloride, diclofenac sodium and chlopheniramine maleate was studied under the same condition, and their possible interference at the elution time of analyte and IS was observed.

The precision and accuracy are evaluated as the relative standard deviation of within or run to run (intra) assay as well as between or day to day (inter) assays. Within and between batch precision were evaluated using previously frozen quality control samples at four different concentrations of $14,345,1728,3200 \mathrm{ng} / \mathrm{mL}$, designated as Low, medium 1 , medium 2 and high concentrations. For between precision, was analysed using six samples of each concentrations for a total of 24 assays on 3 different days using six sets of standard curves. Mean and standard deviation were obtained for the calculated drug concentration over all 3 days and coefficient of variation $(\% \mathrm{CV})$ for all four different levels ( $\mathrm{n}=18$ for each) were determined. For within precision 6 samples from each of four concentrations were assayed with a single calibration curve and coefficient of variation for the calculated drug concentrations were determined.

The Recovery of an analyte in an assay is the detector response obtained from an amount of the analyte added to and extracted from the biological matrix, compared to the detector response obtained for the concentration of the pure authentic standard. Recovery pertains to the extraction efficiency of an analytical within the limits of variability. Recovery of omeprazole from plasma following sample preparation was assessed by comparing the concentration of drug from extracted plasma quality control samples against unextracted quality control samples at different levels. Percentage mean recovery and $\% \mathrm{CV}$ were calculated.

The matrix effect is defined as the combined effect of all components of the sample other than the analyte on the measurement of the quantity. The evaluation of matrix effect is another issue that cannot be underestimated as the calibration strategy to be followed depends on this. It has been proved that cleaner extract such as those resulting from LLE are less affected by matrix effects. Besides the influence of the sample matrix composition on MS and MS/MS responses has often been found to be significant. As a result, strategies based on post extraction and post column infusion have been utilized for assessing matrix effect (Taylor, 2005). To study the effect of matrix on analyte quantification with respect to consistency in signal enhancement / suppression, it was checked in screened 6 different batches (4 for normal plasma, 1 haemolytic and 1lipidemic). Each lot of plasma 
having concentration equivalent to $\mathrm{HQC}$ and LQC were prepared in triplicate and injected. Then it was checked for $\%$ accuracy and precision (\%CV) at HQC and LQC level. This was assessed by comparing back calculated value from the QC's nominal concentration. The deviation of standard should not be more than $\pm 15 \%$, and at least $90 \%$ of the lots at each QC level should be within the acceptance criteria.

Stability experiments were carried out to examine the analyte stability in stock solution and in human plasma samples under different conditions. The long term stability of human plasma was assessed based on the analysis of QC (HQC and LQC) samples that had been prepared and stored at $2-8^{\circ} \mathrm{C}$ for a storage period of 6days and $21 \mathrm{~h}$. The short term stability studies were determined by the QC samples was prepared, stored at $7 \mathrm{~h}$ and $12 \mathrm{~min}$. To assess the room temperature stability of omeprazole in human plasma, quality control samples were allowed to remain at room temperature for $4.30 \mathrm{~h}$ before analysis. Stability was assessed by comparing against the freshly prepared sample with stability sample. Autosampler stability, bench top stability, dry extract stability and freeze extract stability were performed at HQC and LQC using three replicates at each level.

To authenticate the ruggedness of the proposed method, it was done on two precision and accuracy batches. The first batch was analysed by different analysed by different analysts, while the second batch was analysed on two different columns.

Dilution integrity experiment was evaluated by diluting the stock solution prepared as spiked standard at concentration of $9880.73 \mathrm{ng} / \mathrm{mL}$ in the screened plasma for analyte. The precision and accuracy for dilution integrity standards at $1 / 5^{\text {th }}(1976.14 \mathrm{ng} / \mathrm{mL})$ and $1 / 10^{\text {th }}(988.074)$ were determined by analysing the sample against calibration curve standards.

\section{Bioequivalence study design}

This validated method applied to measure the omeprazole concentration in serum samples to evaluate the bioavailability of the single dose of omeprazole $20 \mathrm{mg}$ capsule in 24 healthy volunteers (Kamrun et al., 2009).
The procedures followed while dealing with human subjects were based on International conference on Harmonization, E6 Good Clinical Practice (ICH, E6 GCP) guidelines. The medication was administered under fasting conditions with $250 \mathrm{ml}$ of water. Blood samples were collected at 0.0 (pre dose) $0.25,0.5,0.75$, 1.0, 1.5, 2.0, 2.5, 3.0, 3.5, 4.0, 4.5, 5.0, 5.5, 8.0, $10.0,12.0,24 \mathrm{~h}$ in labelled K2 EDTA vacuettes. After each blood sampling plasma was separated by centrifugation at $3000 \mathrm{rpm}$ for $15 \mathrm{~min}$ and stored at $-80^{\circ} \mathrm{C}$ until further analysis. Serum samples were prepared by liquid -liquid extraction technique.

\section{RESULTS AND DISCUSSION}

Representative chromatograms and mass spectrum of omeprazole and internal standard, extracted under the condition of the assay and of the LOQ $(5 \mathrm{ng} / \mathrm{mL}$ ) (Figure 2) and demonstrate excellent separation of the omeprazole and internal standard with short run times around 1.25 and $1.35 \mathrm{~min}$ respectively. Peak area ratios of omeprazole to IS for the calibration standards were proportional to the concentration of Omeprazole in plasma over the range tested. As the variance increased in proportion to the concentration, the best weighting was $1 / \mathrm{x}^{2}$. The ten standard curves were linear from 5.016 to $4013.02 \mathrm{ng} / \mathrm{mL}$, with a mean equation $0.004749+0.002984$ and an average coefficient of variation were found to be 0.9984 . The selectivity of the method towards endogenous plasma matrix was ascertained in ten batches of human plasma by analysing blanks and spiked plasma samples at LLOQ concentration. No endogenous peaks were observed at the retention time of the analytes for any of the batches.

\section{Recovery}

Six replicates at LQC, MQC and HQC levels (extracted and unextracted) were prepared for recovery determination. The mean percentage recovery at HQC, MQC and LQC levels for omeprazole and IS were 87.54, 86.34, $84.27 \%$ and $86.81,87.24,83.62$ respectively. The $\% \mathrm{CV}$ at all QC levels for omeprazole and internal standard were 6.10 and $4.14 \%$ respectively. 
Table I. Settings for MS - MS detection of omeprazole

\begin{tabular}{cccc}
\hline Source $\left(\mathbf{E S}^{+}\right)$ & Settings & Analyzer & Settings \\
\hline Capillary $(\mathrm{kV})$ & 3.50 & Low mass I resolution & 13.0 \\
Extractor $(\mathrm{V})$ & 4.00 & High mass I resolution & 13.0 \\
RF lens $(\mathrm{V})$ & 0.0 & Ion energy I & 1.0 \\
Source temperature $\left(0^{\circ} \mathrm{C}\right)$ & 120 & Entrance & 2.0 \\
Desolvation temperature $\left(0^{\circ} \mathrm{C}\right)$ & 400 & Exit & 2.0 \\
Cone gas flow $(\mathrm{L} / \mathrm{h})$ & 50 & Low mass II resolution & 13.0 \\
Desolvation gas flow $(\mathrm{L} / \mathrm{h})$ & 800 & High mass II resolution & 13.0 \\
& & Ion energy II & 1.5 \\
& & Multiplier $(\mathrm{V})$ & 650 \\
& & Collision gas pressure (mbar) & $3.5 \mathrm{e}^{-3}$ \\
\hline
\end{tabular}

a.
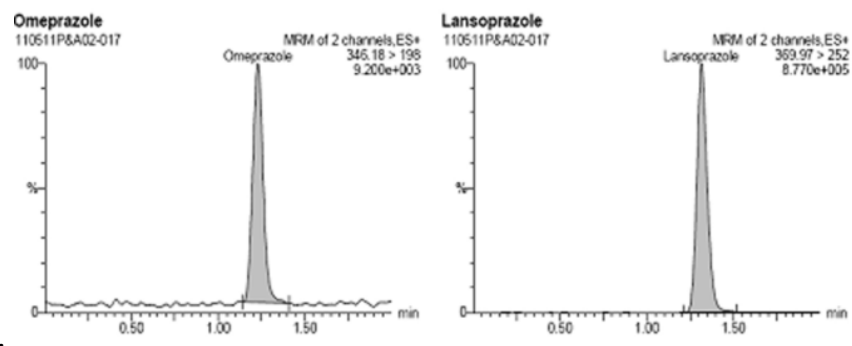

b.
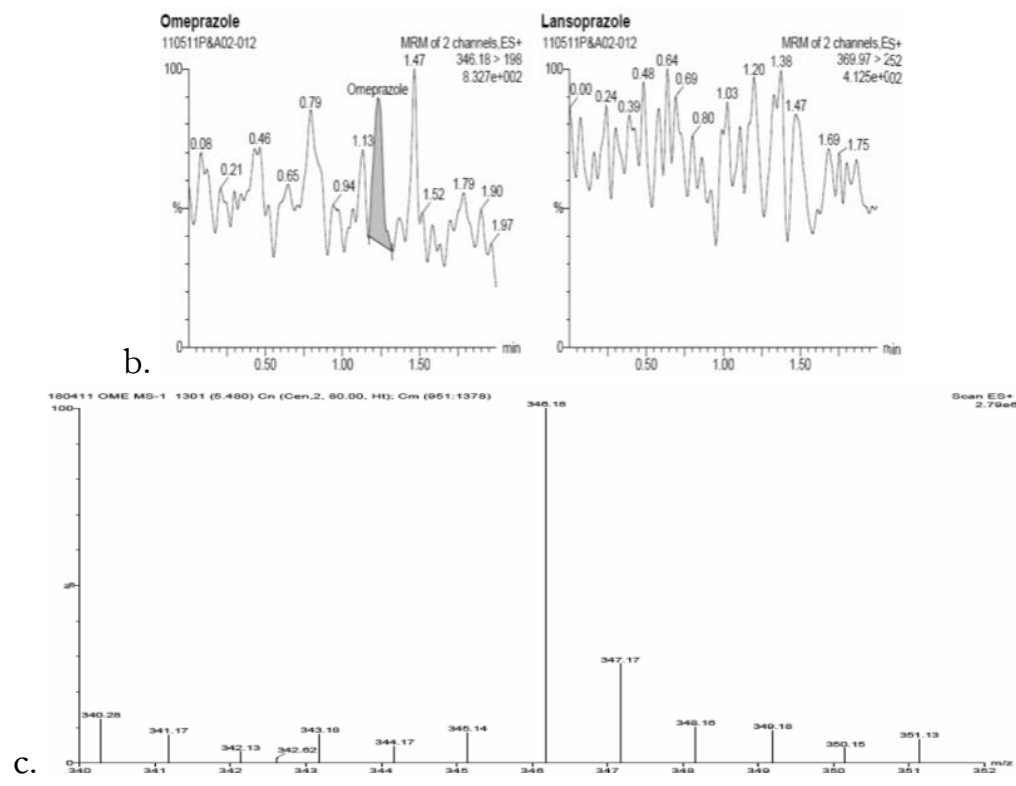

Figure 2. a Mass chromatograms of omeprazole and lansoprazole, b Mass Spectrum of omeprazole and lansoprazole, c Mass spectrum of omeprazole

Within and between batch accuracy and precision

The within and between batch accuracy and precision was determined in four batches at LQC, MQC1, MQC2 and HQC levels with six replicates for each batch. Precision (\%CV) for within batch and between batches ranged from 0.59 to $8.92 \%$ and 2.49 to $5.17 \%$ for analyte. The accuracy results for within batch and between batches were ranged from $91.56 \%$ to $99.01 \%$ and 93.32 to $98.0 \%$ for analyte respecttively, at all quality control levels (Table II). 
Table II. Within- and between batch Precision/Accuracy for omeprazole

\begin{tabular}{lrrcrc}
\hline \multicolumn{1}{c}{ QC ID } & $\begin{array}{c}\text { Nominal Conc } \\
(\mathbf{n g} / \mathbf{m L})\end{array}$ & $\mathbf{n}$ & $\begin{array}{c}\text { Mean Observed Conc } \\
(\mathbf{n g} / \mathbf{m L})\end{array}$ & $\mathbf{\%} \mathbf{C V}$ \% Accuracy \\
\hline Within batch & & & & & \\
HQC & 3266.69 & 6 & 3213.45 & 2.13 & 98.37 \\
MQC1 & 1764.04 & 6 & 1746.56 & 0.59 & 99.01 \\
MQC2 & 352.80 & 6 & 346.05 & 2.63 & 98.09 \\
LQC & 14.95 & 6 & 13.69 & 2.50 & 91.56 \\
LLOQ & 5.14 & 6 & 4.70 & 5.71 & 91.34 \\
Between Batch & & & & & \\
HQC & 3266.69 & 18 & 3183.52 & 2.97 & 97.45 \\
MQC1 & 1764.04 & 18 & 1725.63 & 2.49 & 97.82 \\
MQC2 & 352.80 & 18 & 345.93 & 5.17 & 98.05 \\
LQC & 14.95 & 18 & 13.95 & 3.42 & 93.32 \\
LLOQ & 5.14 & 18 & 4.78 & 10.24 & 92.93 \\
\hline
\end{tabular}

Table III. Matrix Effect Data of Omeprazole in Six Different Lots of Human Plasma at HQC and LQC Level

\begin{tabular}{ccccc}
\hline \multirow{2}{*}{ Lot no } & \multicolumn{2}{c}{ HQC 3266.7* } & \multicolumn{2}{c}{ LQC 14.95* } \\
\cline { 2 - 5 } & $\begin{array}{c}\text { Calculated Conc. } \\
\text { (ng/mL) }\end{array}$ & $\begin{array}{c}\text { \% } \\
\text { Accuracy }\end{array}$ & $\begin{array}{c}\text { Calculated Conc. } \\
\text { (ng/mL) }\end{array}$ & $\begin{array}{c}\text { \% } \\
\text { Accuracy }\end{array}$ \\
\hline 1 & 3025.19 & 92.60 & 13.62 & 91.10 \\
2 & 3224.07 & 99.30 & 14.69 & 98.26 \\
3 & 3133.16 & 95.91 & 13.04 & 87.22 \\
4 & 3398.86 & 104.04 & 14.20 & 94.98 \\
5 & 3308.31 & 101.27 & 14.47 & 96.89 \\
6 & 3130.99 & 95.84 & 12.47 & 83.41 \\
Mean & & 98.16 & & 51.97 \\
SD & & 4.17 & & 6.82 \\
\%CV & 5.62 & & \\
*Nominal concentration (ng/mL) & & & \\
\hline
\end{tabular}

\section{Ion suppression, matrix effect}

Matrix effect may arise due to co-elution of some unintended components present in biological samples or which are added as a part of analysis. These components may result in ion suppression/enhancement, decrease/ increase in sensitivity of analyte over a period of time, increase baseline, imprecision of data, drift in retention time and distortion or tailing of a chromatographic peaks. (Matuszewski et al., 2003; Dams et al., 2003) The precision $(\% \mathrm{CV})$ values for HQC and LQC samples were observed were 4.32 and $5.46 \%$, while the accuracy found at this levels were 96.46 and $91.18 \%$ for analyte (Table III).

Stock solutions for long- term and short - term stability of the analyte and internal standard were stable at room temperature for minimum period of $21 \mathrm{~h}$ between $2-8^{\circ} \mathrm{C}$ for 6 days, respectively. Analyte in control human plasma (bench top) at room temperature were stable at least $7 \mathrm{hr}$ at $25^{\circ} \mathrm{C}$ and for a minimum of four freeze - thaw- cycles. Spiked plasma samples stored at $2-8^{\circ} \mathrm{C}$ for long stability experiment were stable for a minimum of 6day. Dry extract stability of the spiked quality control samples stored at $-28^{\circ} \mathrm{C}$ was determined up to $66 \mathrm{~h}$. Auto sampler stability of the spiked quality control samples maintained at $5^{\circ} \mathrm{C}$ was determined up to $67 \mathrm{~h}$. Different stability experiments in plasma and the values for the precision and percentage change (Table IV). 
AN LC- MS/MS Method For The Determination

Table IV. Stability Data of omeprazole in Human plasma under various storage conditions ( $\mathrm{n}=6$ )

\begin{tabular}{lrrr}
\hline \multirow{2}{*}{ Storage Conditions } & Mean comparison & \multicolumn{2}{c}{ Calculated Concentration (ng/mL) } \\
\cline { 3 - 4 } samples & Mean stability & \% mean sample stability \\
\hline Auto sampler stability & & & \\
HQC & 3153.69 & 3403.28 & 107.91 \\
LQC & 15.03 & 15.96 & 106.19 \\
Bench Top Stability & & & \\
HQC & 3208.09 & 3202.75 & 99.83 \\
LQC & 14.47 & 15.17 & 104.87 \\
Freeze thaw stability & & & 105.81 \\
HQC & 3153.69 & 3346.69 & 98.43 \\
LQC & 15.03 & 16.13 & \\
Wet extract stability & & & 97.43 \\
HQC & 3208.09 & 3125.64 & \\
LQC & 14.47 & 13.89 & 106.81 \\
Dry extract stability & & & 105.74 \\
HQC & 3153.69 & 3368.50 & \\
LQC & 15.03 & 15.89 &
\end{tabular}

Table V. Mean pharmacokinetic parameter following Oral administration of $20 \mathrm{mg}$ tablet formulation (Test and Reference) of Omeprazole in 20 healthy human subjects

\begin{tabular}{lll}
\hline Parameter & Test Formulation & Reference formulation \\
\hline $\mathrm{T}_{\max }(\mathrm{h})$ & 2.60 & 2.08 \\
$\mathrm{C}_{\max }(\mathrm{ng} / \mathrm{ml})$ & 1011.53 & 1335.46 \\
$\mathrm{t}_{1 / 2}(\mathrm{~h})$ & 1.27 & 1.05 \\
$\mathrm{AUC}_{0-\infty}$ & 3240.01 & 3480.69 \\
$\mathrm{AUC}_{0-\mathrm{t}}$ & 3252.80 & 3456.04
\end{tabular}

$\mathrm{T}_{\max }$ - Time of the $\mathrm{C}_{\max }, \mathrm{C}_{\max }-\mathrm{Maximum}$ observed concentration, $\mathrm{AUC}_{0-\infty}$ - area under the concentration time curve extrapolated to infinity, $\mathrm{AUC}_{0-t-\text { area }}$ under the concentration curve from time zero to the last measurable concentration, $\mathrm{t}_{1 / 2}$ - elimination half -time.

For ruggedness study, the precision and accuracy of calibration curve standards and LLOQ were between $1.43-5.25 \%$ and $96.14-$ $109.45 \%$ for analyte, which is within the acceptance criteria. For both experiments the precision and accuracy for LLOQ, all QC samples ranged from $1.98-11.53 \%$ and 97.89 102.73 for analyte, which are within the acceptance limit of $15 \%$ in precision and 85.0$115.0 \%$ in mean accuracy.

The dilution integrity experiment was performed with an aim to validate the dilution test to be carried out analyte concentration above the upper limit of quantification (ULOQ), which may be encountered during real subject sample analysis. The precision of dilution integrity of $1 / 5^{\text {th }}$ and $1 / 10^{\text {th }}$ dilution were found to be 0.75 and $4.17 \%$ respectively for analyte, while the accuracy results were found to be 98.08 and $94.49 \%$ respectively for analyte. The results were within the acceptance limit of $15 \%$ for precision $(\% \mathrm{CV})$ and 85.0 $115.0 \%$ for accuracy.

\section{Application of the method in Human subjects}

The analytical method had been applied successfully for the analysis of samples from several pharmacokinetic studies. The assay of omeprazole in healthy volunteers in the age group of 20-45 years. Figure 3 shows the mean plasma concentration time profile following $20 \mathrm{mg}$ of dosing to 30 human subjects under fasting conditions up to $24 \mathrm{~h}$. In all samples 
including the calibration and quality control samples with volunteers samples were run and analysed over a period of 10days. The precision and accuracy for calibration and QC samples were well within acceptable limits. The pharmacokinetic parameters like plasma concentration maximum $\left(\mathrm{C}_{\max }\right)$, area under the plasma concentration -time curve from zero hour to infinity $\left(\mathrm{AUC}_{0-i n f}\right),\left(\mathrm{AUC}_{0-t)}\right)$ area under the concentration time curve extrapolated to infinity, time point of plasma concentration maximum ( $\left.\mathrm{T}_{\max }\right)$ were calculated. The pharmacokinetic parameters obtained for the test and reference formulation (Table V).

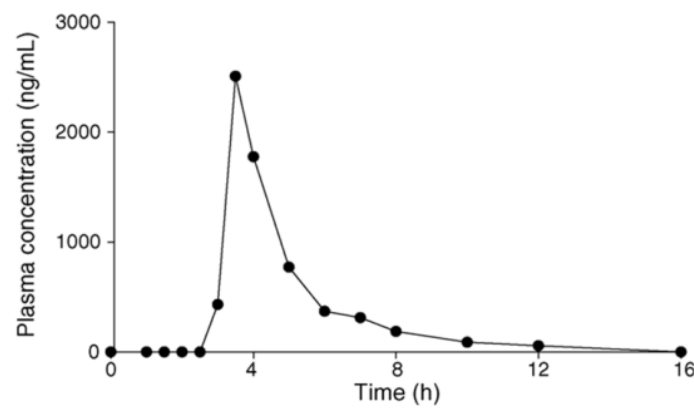

Figure 3. The mean plasma concentration time curve

The aim of the work was to develop and validate an assay for omeprazole in human plasma, which will be suitable for clinical pharmacology purposes. For clinical pharmacology studies, a simple and rapid extraction step is necessary, which is why liquid-liquid extraction was chosen. This extraction method was also used by Kang et al., 1999; Wang et al., 2004; Desmet et al., 2010; but with higher limit of quantification by Bharathi et al., 2009A solid phase extraction of 90\% efficiency was proposed by Woolf et al., 1998; Kanazawa et al., 2004 but it is more expensive than liquid-liquid extraction. The simple protein precipitation extraction procedure was investigated but proved to be unacceptable due to interference and low sensitivity. Liquid-liquid extraction was then investigated using different extraction buffers like, $0.5 \% \mathrm{NH}_{3}, 10 \mathrm{mM}$ ammonium acetate buffer $\mathrm{pH}$ 8.0, ammonium acetate $\mathrm{pH} 10.0$ and $0.1 \%$ formic acid. Among these buffers $10 \mathrm{mM}$ ammonium acetate (pH10.0) buffer was found to be showing reproducible results. $50 \mu \mathrm{L}$ instead of $100 \mu \mathrm{L}$ extraction buffer and $2 \mathrm{~mL}$ TMBE were used.
In general HPLC coupled to MS/MS instruments is used to monitor specific mass transitions so analytes can be quantified free of interferences even in poorly resolved chromatographic peaks. Compounds are usually detected in positive mode from their $[\mathrm{M}+\mathrm{H}]^{+}$peaks. Electrospray is the ionization source to be combined with triple quadrupole analyser. Full-scan positive-ion spectra of omeprazole and internal standard predominantly the protonated molecular ion at $\mathrm{m} / \mathrm{z} 346.18$ and 369.97 respectively. The product ion mass spectra of these protonated molecular ions (Figure 2) show the presence of one predominant ion for each compound at $\mathrm{m} / \mathrm{z}$ and for omeprazole and internal standard respectively. Several columns were tested to assay omeprazole, such as Kromosil luna (100X $\left.4.6 \mathrm{~mm}, 5 \mu, \mathrm{C}_{18}\right)$, Grace smith (150 X 4.6mm, $\left.5 \mu, C_{18}\right)$. In these two columns response peak shape was good, column pressure was more and lower level repeatability was bad. Phospher

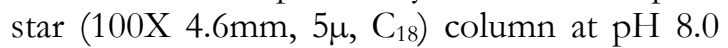
was evaluated and found to provide adequate peak shape and resolution for the analysis of omeprazole. Omeprazole when prepared in solution with a $\mathrm{pH}$ of less than 8 has been reported to degrade rapidly. Therefore, in order to maximize analyte stability, a chromatographic system that utilized a mobile phase with a $\mathrm{pH}$ greater than 8 was required. Several mobile phases were tested with the chosen column methanol/ammonium acetate (pH 8.0) mixture that was associated with a bad peak resolution, ammonium bicarbonate and ammonium formate buffer / methanol in different proportions that provided a retention time superior to 10 minutes. Ammonium bicarbonate $5 \mathrm{mM}$ buffer $\mathrm{pH}(8.0) /$ acetonitrile are needed to maintain omeprazole (without peak tailing factor) and ensure symmetry and thickness of Omeprazole peak. Therefore bicarbonate buffer was selected rather than the acetate and formate buffers used by other authors authors (Vittal et al., 2009; TsingHua, 2004 Kanazawa et al., 2004) since the possibility of precipitation in HPLC system was less likely. The LLOQ found with our method $(5 \mathrm{ng} / \mathrm{mL})$ is lower than the LOQ of other quantification LC-MS/MS methods (Vittal et al., 2009; Desmet et al., 2010 Wang et al., 2004 ) but greater than the HPLC quantification methods 
(Bharathi et al., 2009; Zaraghi et al., 2006) . Limit of quantification of the methods using HPLC $20.6 \mathrm{ng} / \mathrm{mL}$ with plasma sample and a liquid/liquid step for (Bharathi et al., 2009) $10 \mathrm{ng} / \mathrm{mL}$ with plasma sample and a liquid /liquid extraction step for Zarghi et al. (2006) However, the LOQ of $5 \mathrm{ng} / \mathrm{mL}$ is sufficient to quantify plasma omeprazole concentration in healthy volunteers according to previously published papers (Kamrun et al., 2009; Liu et al., 2012).

Carryover evaluation was performed in each analytical run so as to ensure that it does not affect the accuracy and precision of the proposed method. There was no carry over observed during autosampler carryover experiment. Also no enhancement in the response was observed in the double blank after subsequent injection of highest calibration standards at the retention time of omeprazole and internal standard respectively. Moreover, no ghost peaks appeared during the analysis of blank samples. The retention time for omeprazole and internal standard were at 1.25 and $1.35 \mathrm{~min}$ respectively. None of the concomitant medications considered showed interfering signals at the retention time of omeprazole or internal standard. This demonstrates that the method is highly selective and free from interference due to matrix components and other prescribed medications. The assessment of matrix effect constitutes an important an integral part of validation for quantitative LC-MS-MS there, the matrix effect for the intended method was assessed by using chromatographically screened human plasma. From the result of analysis (Table III) it indicated thus the method was rugged and gave accurate and consistent results when applied to subject sample analysis. In comparison with the previously developed methods, the present method offers an undoubted advantage in terms of overall analytical performance.

\section{CONCLUSION}

A simple and sensitive rapid LCMS/MS assay for the quantitation of omeprazole in small volume of plasma was developed and validated. The method was shown to be specific, accurate, precision and reproducible. The method can be applied to the pharmacokinetic evaluation and also can be applied for further pharmacokinetic characterization of Omeprazole.

\section{ACKNOWLEDGMENT}

The authors wish to thank for the support received from The Erode college of Pharmacy, Erode, Tamilnadu, India, for providing literature survey, The Dr.M.G.R. Medical University, Chennai, India. Authors are grateful to Aurbindo Pharmaceutical Pvt, Ltd, Hyderabad, India for providing clinical samples and support from Pharmacokinetic data.

\section{REFERENCES}

Antonio Checa, Ramon Oliver, Santiago Hernandez Cassou, Javier Saurina, 2009. Determination of HIV drugs in biological matrices: A review. Anal. Chimica Acta.647:1-13.

Bharathi DV., Hotha KK., Jagadeesh B., Chatki PK., Thriveni K., Mullangi R, Naidu A., 2009. Simultaneous estimation of four proton pump inhibitors-lansoprazole, omeprazole, Pantoprazole and rabeprazole development of a novel generic HPLC-UV method and its application to clinical pharmacokinetic study. J. Bio. Med. chromatogr. 23(7): 732 739.

Dams R., Huestis MA., Lambert WE., Murphy CM., 2003. Matrix effect in Bio-analysis of liquid drugs with LC-MS/MS: influence of ionization type, sample preparation and bio fluid. J. Am. Soc. Mass. 14(11):1290-94.

De Smet J., Boussery K., De Cock P., De Paepe P., Remon JP., Van Winckel M., Van Boussery J., 2010. A Bio-analytical hydrophilic interaction LC- MS/MS method for the simultaneous quantification of omeprazole and lansoprazole in human plasma in support of a pharmacokinetic omeprazole study in children. J. Sep. Sci. 33(6-7): 939-947.

Furuta T., Takashima M., Shirai N., Xiao F., Hanai H., Ohashi K., Ishizaki T., 2000. Cure of refractory duodenal ulcer and infection caused by Helicobacter pylori by high doses of omeprazole and amoxicillin in a homozygous CYP2C19 
extensive metabolizer patient. Clin. Pharmacol. Ther. 67: $684-689$.

Guidance for Industry: ICH E6 Good Clinical Practice, US Department of Health and Human services, Food and Drug Administration Centre for Drug Evaluation and Research (CDER), Centre for Biologics Evaluation and Research (CBER), April 1996.

Guidance for Industry, Bio analytical method validation, US Department of Health and Human services, Food and Drug Administration Centre for Drug Evaluation and Research (CDER), Centre for Veterinary Medicine(CBER), May 2001.

Jian Liu, Jian-zhong Shentu, Li-hua Wu, Jing Dou, Qi-yang Xu, Hui-li Zhou, Guo-lan Wu, Ming-zhu Huang, Xing-jiang Hu, Jun-chun Chen.2012. Relative bioavailability and pharmacokinetic comparison of two different enteric formulations of omeprazole. J. Zhejiang. Univ. Sci B. 13(5): 348-355.

Kamrun Nahar, Jafreen Jamal Joti, Md Ashik Ullah, Ahasanul Hasan, Mohammad Abul Kalam Azad, Abul Hasnat, 2009.A Simple RP-HPLC Method for the Determination of Omeprazole in Human Serum and Urine: Validation and Application in Pharmacokinetic Study. Dhaka University J. Pharma. Sci. 8(2):

Kanazawa H., Okada A., Matsushima Y., Yokota H., Okubo S., Mashige F., Nakahara K., 2004. Determination of omeprazole and its metabolites in human plasma by liquid Chromatography-mass spectrometry. J. Chromatography. 949(1-2): 1-9.

Kang WK., Kim DS., Kwon KI., 1999. Advanced method for determination of omeprazole in plasma by HPLC. Arch. Pharma. research. 22(1); 86-88.

Matuszewski BK., Constanzer ML., ChavezEng CM., 2003. Strategies for the Assessment of Matrix effect in Quantitative Bio analytical methods based on HPLC- MS/MS. J Anal. Chem. 75(13):3019-30.

Rambla-Alegre M., Esteve-Romero J., CardaBroch S.. 2009. Analysis of omeprazole and its main metabolites by liquid chromatography using hybrid micellar mobile phases. Anal. Chim. Acta. 633(2):250-6

Rang HP., Dale MM., Ritter JM., Flower RJ. 2007. Pharmacology. $6^{\text {th }}$ ed. Elsevier, Philadelphia, Churchill Livingstone, pp. 295-96.

Stenhoff H., Blomqvist A., Lagerström PO.,1999. Determination of the enantiomers of omeprazole in blood plasma by normal-phase liquid chromatography and detection by atmospheric pressure ionization tandem mass spectrometry. J. Chromatogr. B. 734: 191.

Shiohira H., Yasui-Furukori N., Tateishi T., Uno T..2011. Chiral assay of omeprazole and metabolites and its application to a pharmacokinetics related to CYP2C19 genotypes. J. Chromatogr. B Analyt. Technol. Biomed. Life Sci. 879(24):2465-70

Taylor PJ., 2005. Matrix effects: the Achilles heel of quantitative high-performance liquid chromatography-electrospraytandem mass spectrometry. Clin Biochem.38(4):328-34

TsingHua. 2004. Determination of relative bioavailability of omeprazole entericcoated capsules in healthy volunteer. Chinese.J. hospital pharmacy. $2002-08$.

Vittal S., Ganneboina R., Layek B., Rived RK., Hotha KK., Bharathi DV., Mullangi R., 2009. Highly sensitive method for the determination of omeprazole in human plasma by liquid Chromatographyelectrospray ionization tandem mass spectrometry: application to a Clinical pharmacokinetic study. Biomed. chromatogram. 23(4): 390-6.

Wang J., Wang Y., Fawcett JP., Wang Y., Gu J. 2004. Determination of omeprazole in human plasma by liquid chromatography - electrospray quadrupole linear ion trap mass spectrometry. J. Bio med anal. 39 (3-4): 631-635.

Woolf EJ., Matuszewski BK., 1998. Simultaneous determination of omeprazole and 5'-s hydroxyomeprazole in human plasma by liquid chromatography-tandem mass 
AN LC- MS/MS Method For The Determination

spectrometry. J. Chromatogr A 828(1-2): 22.

Yim DS., Jeong JE., Park JY., 2001. Assay of omeprazole and omeprazole sulfone by semi-microcolumn liquid chromatography with mixed-function precolumn. J Chromatogr B Biomed Sci Appl.754(2):48793
Zarghi A., Foroutan SM., Shafaati A., Khoddam A., 2006. Determination of omeprazole in blood plasma by using HPLC. Aræneimittelfor schung 2006;56(6) 382-6.

Figure 3 The mean plasma concentration time curve 